\title{
iLeger: A Web Based Application for Participative Elections
}

\author{
Artur Afonso Sousa ${ }^{1}$, Pedro Agante ${ }^{2}$, and Luís Borges Gouveia ${ }^{3}$ \\ ${ }^{1}$ Polytechnic Institute of Viseu, Viseu, Portugal \\ ajas@di.estv.ipv.pt \\ ${ }^{2}$ Libertrium, Viseu, Portugal \\ pedroagante@libertrium.com \\ ${ }^{3}$ University Fernando Pessoa, Porto, Portugal \\ lmbg@ufp.edu.pt
}

\begin{abstract}
Leger is a Web Application that seeks to concentrate, in a single place, the stakeholders in a political election allowing multi-directional and structured communication between them. Using a citizen and candidate centered approach; iLeger supports collaborative interaction with the purpose of fostering communication, deliberation and participation. This paper aims at presenting the main functionalities of this Web application, as well as the results from a case study about the Portuguese Presidential Elections held in 2011.
\end{abstract}

Keywords: Elections, Campaigning, eDemocracy, eParticipation, Deliberation.

\section{Introduction}

The current economic and social context calls to civic intervention and stimulates the search for solutions and answers. Recently we have been witnessing a growth in the adoption of Information and Communication Technology (ICT) and the widespread access to the Internet. Following this trend, the field of public participation [1] has seen a growing integration of ICT and the Internet, leading to the concept of electronic participation - eParticipation [2]. The potential of technology to increase public participation has been a topic of debate in recent years. Although there are skeptics [3], we believe, as [4, 5] that the Internet-based technologies have the potential to change and improve how the stakeholders interact with each other in the democratic process.

The research area of eParticipation is still in its infancy [6]. Although there are already some initiatives [7], they generally tend to serve a specific purpose and scope [8]. This article describes a Web application, iLeger, specifically designed to gather during the election period, voters and candidates in an election in a shared deliberative space. With this application it is intended to contribute to close the communication gap identified between these two key stakeholders.

ILeger is integrated into the project Liberopinion (http://www.liberopinion.com) which aims to create a technology platform in the field of eDemocracy and social networks, with emphasis on interaction between users. Currently, the platform 
Liberopinion consists of two applications, the one described in this paper, iLeger, and Governmeter, which is intended to monitor the performance of governmental activity. In summary, Governmeter is a web application based on the principles of eParticipation, specifically designed to monitor and discuss, objectively and independently, the government activity and new laws at national, regional or local level. In a first stage, Governmeter is mainly focused on three aspects: the evolution of conjuncture indicators, the government objectives and government measures.

In more detail, iLeger combines in a neutral and civilized single space the key stakeholders in an election, the candidates and citizens, and promotes multidirectional communication between them. Interaction and collaboration is supported through questions, answers, suggestions, comments, votes and live debates. ILeger was recently tested in the Election of the Chairman of the Portuguese Medical Association (http://om.ileger.sapo.pt) and used in partnership with the largest Portuguese Web portal (SAPO - http://www.sapo.pt), property of Portugal Telecom, in the Portuguese Presidential Election of 2011 (http://presidenciais.ileger.sapo.pt).

In the use of Web communication tools to reach voters on general elections, there has been major investments by all political parties and corresponding candidates on either social networks such as Facebook, Twitter, MySpace, or on dedicated websites through which the candidates attempt to present their positions and their electoral program. This combined with the traditional television debates, ads, and rallies through the country, make up for the most part of the political campaign.

If we consider now the citizen perspective and the information gathering process that precedes the voting decision, we have two main sources of information. The first, which might be called passive, consists of watching the news or debates and speeches by the candidates in the TV or radio, as well as the analysis by political experts. In the second, an active one, usually the citizen consults electoral program of the different candidates, typically on the candidates' website, or at best consult other media websites which aggregates this information and provides a comparison of the candidates position on each topic or issue.

After observing the traditional type of political communication and media coverage, important questions emerged: in the era of widespread social interaction can this be the best method for citizens to decide for a particular candidate? How can we, as individuals and as a community, make sure that our most important problems are being correctly identified and directly addressed by the different candidates? Is there an efficient way to take advantage of the collective knowledge and ideas of the community, to help the candidates draft the solutions to these problems? Finally, how to find the best candidate for us, both as an individual and as a community?

In an attempt to answer such questions, we realized that there must be a better way to manage the citizen-candidate interaction. We consider that it would be useful to have an application that unites, in a single, neutral and civilized place, the stakeholders in the electoral process so as to allow multidirectional communication between them. Such perspective takes into consideration the need to provide an eparticipation citizen and candidate centered tool. This would enable, on the one hand, the citizens to become clarified on the most important questions and problems of society and, on the other hand, the candidates to be aware of the citizens' ideas and the main concerns of the community in different governance topics (education, health, economy, justice, and so on). 
This paper is structured as follows: Section 2 describes the major functionalities of the iLeger Web application, while section 3 presents the results from a case study about the Portuguese Presidential Elections held in January 2011. Section 4 contains topics for future work. Finally, the section 5 closes the paper by presenting conclusions.

\section{The iLeger Web Application}

Considering either general online tools such as blogs, Internet campaign sites of the political parties, email, email newsletters, or more traditional media covering TV broadcasts, debates, telephone calls, door-to-door contact or town hall speeches, they are mostly concentrated in unidirectional communication and do not support an efficient, scalable communication process based on all stakeholders' goals and needs.

Before the 2008 presidential TV debate between Obama vs. McCain, the TV station prompted the population to submit their questions to the candidates. From the pool of questions, a selection would be made and the resulting questions would be presented to the presidential candidates, in addition to the ones presented by a panel. Six million submissions were received by means of email, comments in the TV station website, telephone calls, among others. The number of questions formally submitted to the official website for this purpose, Mydebates.org, was about 25,000 [9]. This is one example of the willingness and initiative of citizens when prompted to interact with candidates, especially when they are prompted to bring forward their own problems and concerns to their potential representatives.

Moreover, it was found that three-quarters (74\%) of Internet users went online during the 2008 US election to take part in, or get news and information about the campaign [10]. This represents 55\% of the entire US adult population, and marks the first time the Pew Internet \& American Life Project has found that more than half the voting-age population used the Internet to connect to the political process during an election cycle. The Internet has therefore emerged as an ubiquitous support means used by citizens to clarify the issues important for their voting decision.

Several online initiatives have been made available to help citizens clarify the issues and proposals by the different candidates. A known approach presents a questionnaire to citizens covering different issues, make a statistical comparison with the candidates' stances and derive the candidate that best matches the user answers [11]. This does not allow the citizen to communicate and submit questions to any of the candidates. The questions formulated are based in the electoral program as defined by each candidate and does not provide any basis for interaction. Other websites comparing the different candidates' proposals in several topics also exist [12].

On the other hand, another approach seeks to close the communication gap between citizens and politicians [13]. The list of political representatives, as well as election candidates, is displayed and it is possible for citizens to submit questions and for the politicians to answer. However, the website is designed around each political representatives and doesn't seem to provide neither a scalable solution when the number of questions increase, nor a direct comparison of candidates' answers to the same question and debate around the question and answers. 
ILeger is a Web Application designed and developed from the ground up to meet the needs and stakeholders goals in the electoral process, considering the two major groups of citizens and candidates. As illustrated in Figure 1, it consists of five main areas: questions from the citizens and corresponding answers from the candidates, proposals and ideas from the community, citizen surveys, the candidates' electoral program and finally live debates.

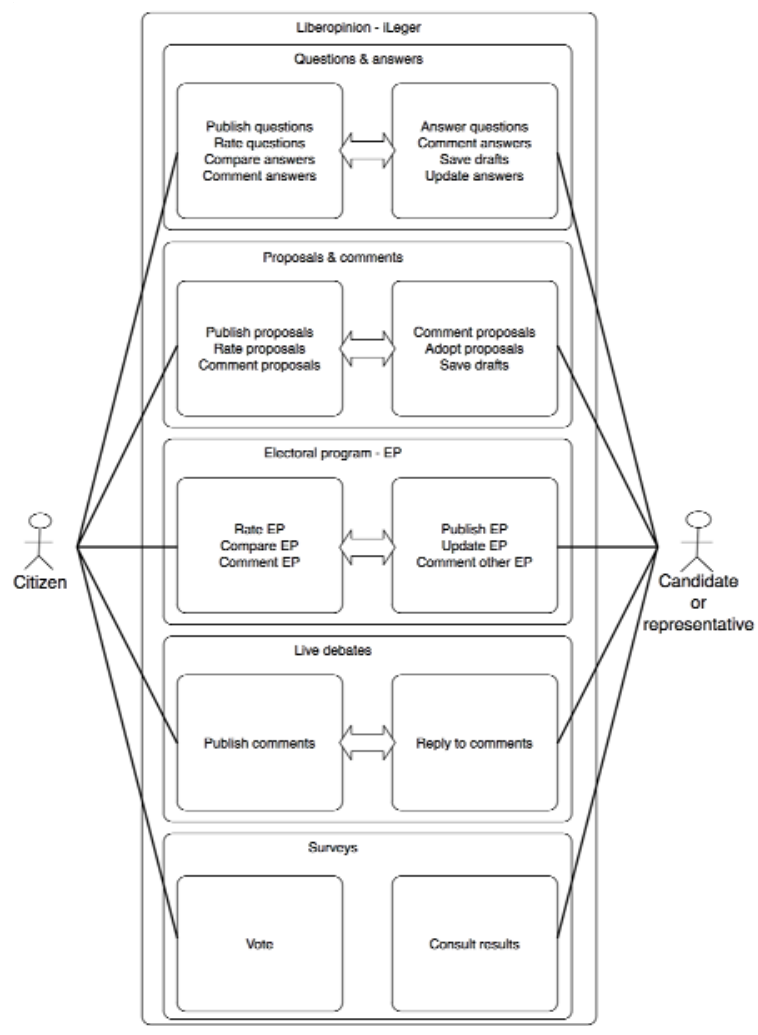

Fig. 1. Functional structure of iLeger

We believe that online social networks will be increasingly important for communities and citizens. We considered from very important to provide iLeger platform with social networking features at an early stage. Therefore, a registered user can follow other users registered on the platform, and see all the questions, suggestions and comments made by those users.

Seamless integration of the platform with existing social networks is also of paramount importance [14]. For this reason, and as a first step in that direction, it is possible for a citizen to publish his questions and proposals to Twitter and Facebook directly from the platform. Thus provide a more open interface to foster participation of enhanced opinion voice, as it augments the scope and potential impact of each individual's participation. 
In relation to user registration and access to the platform, by default, each user must be previously registered on the platform and have to login to take advantage of key capabilities such as submit questions, suggestions, comments and voting. If the user is not logged in, it is only possible to view the contents of the application. Nevertheless, it is possible to configure iLeger for different settings so as to enable interaction by non-registered users. For example, there is a setting in which nonregistered users can vote but cannot introduce content and other that allows voting and content introduction. In the latter configuration, the only limitation to unregistered users is the lack of email notifications and additional features known from social networks such as following user and the access to a public profile.

By default, all the content submitted by citizens is subject to moderation [15] according to the platform terms of use. When the user registers in the platform, the user must accept these terms of use. The actions of the candidates, on the other hand, are not moderated. The platform is configured to support different settings for moderation. For example, it is possible to publish directly all content, i.e. disable moderation, or moderate only entries denounced by the platform users. In order to encourage participation, citizens are permitted to request anonymity for all content submitted to the platform.

Currently, iLeger can be configured regarding the type of interaction of candidates. iLeger is foreseen to be used in two scenarios: with or without interaction by candidates. With interaction, the candidates have an access account and are responsible for the introduction of content, allowing direct communication with other users. In the absence of candidate interaction, iLeger can still be used to identify key issues and suggestions from voters, as well as their views on the key issues about the election.

In the following sections, the iLeger platform is described in more detail.

\subsection{Questions from the Citizens}

ILeger can be configured to be used with or without direct interaction of the candidates. Without direct interaction this area of iLeger serves primarily to generate a TOP of questions that will be used to fuel the discussion directly with the candidatures. In a first phase, users are invited to submit questions to the candidates and vote on its relevance during a predetermined period of time. Then the TOP questions will be asked to representatives of the candidatures during live debates. In a more interactive mode, the candidates have an access account to iLeger and this area allows them to directly answer questions from voters and participate in debates with other candidates and citizens.

The voters are given the chance to raise questions in previously defined topics simultaneously to all candidates whose answers could help them decide which candidate to vote for. Questions and corresponding answers are published for all too see, comment and rate.

One major result of the citizen centered design is the possibility to compare side by side the answers of different candidates to the same question. The voter can thus have a better overview on the substance of the answer, helping to clarify remaining doubts about whom to vote. On the other hand, for answering the questions, the candidates have a dedicated interface in the application where they can write the reply and 
automatically publish it. In order to help the candidate draft a reasoned and thorough answer, it possible to save the answer as a draft to be finished and published at a later stage.

ILeger was designed in such a way that the candidates can answer every question using the same kind of interface, providing a consistent user experience. For instance, when browsing through the list of questions as a citizen would do, the candidate can press the button "answer" to start typing the text or even bookmark a question for a later answer. In order to foster deliberation [16], all interactions between citizens and candidates are associated with a specific comment area. The comments section associated with every question and answers allows easy and intuitive follow up of discussion threads by implementation of a "reply to comment" mechanism. This way, the candidates have a greater insight about main concerns and opinions from citizens and are given the opportunity to present in greater detail their points of view.

\subsection{Proposals and Ideas from the Community}

In this area, the citizens can provide their own ideas and solutions to problems on different topics of governance so that the candidates can benefit from the collective knowledge of the general and specialized community. The interaction model used in this area is similar to the questions: the proposals can be listed per topic; rated for degree of support among the community; and commented on. This way, it provides a community-based mechanism for enhancing and identifying the best proposals ranging different topics such as Health Care, Education, Economy, among many others possible, which can serve as a source of ideas for candidates and political parties (this occurs in a particular time when they are asked for solutions to the national or local themes / issues).

In the configuration without candidate interaction this area allows identification of the best proposals from the citizens, the most voted by the community, in the various areas of governance. Some of these proposals may subsequently be used in direct discussions with representatives of the candidatures to get to know their views.

On the other hand, in case iLeger is configured for direct interaction by the candidates, they are also given the chance to provide, in this area, feedback on the proposals by leaving comments on a text area designed for this purpose. As in the section dedicated to the candidate's answers to the questions, the comments by the candidates are placed side-by-side for an easy comparison by the reader. To foster constant feedback to the candidate and to provide means to ascertain the community reaction to the comments, all these entries are also subject to rating by the citizens.

In order to encourage citizen participation and, possibly to recognize and benefit from the good ideas coming from the community, the candidates can point out by a special icon any proposals that were fully or partially incorporated into the candidate's electoral program. This feature provides, on the one hand, an incentive for the citizens to present their proposals and, on the other hand, a means for the candidates to collect ideas to their programs and emphasize them, a nice feature that encourages a more collaborative political process.

In summary, iLeger provides an additional channel created to foster community participation and communication between the citizens and candidates with the 
objective of identifying the best solutions to the problems faced by society stemming from the community.

\subsection{Citizen Surveys}

Similarly to known e-consulting approaches [17], in this section, several questions are presented to the citizens about key issues about the election. Through a simple and intuitive interface, as shown in Figure 2, the electors are invited to give their opinion through voting.

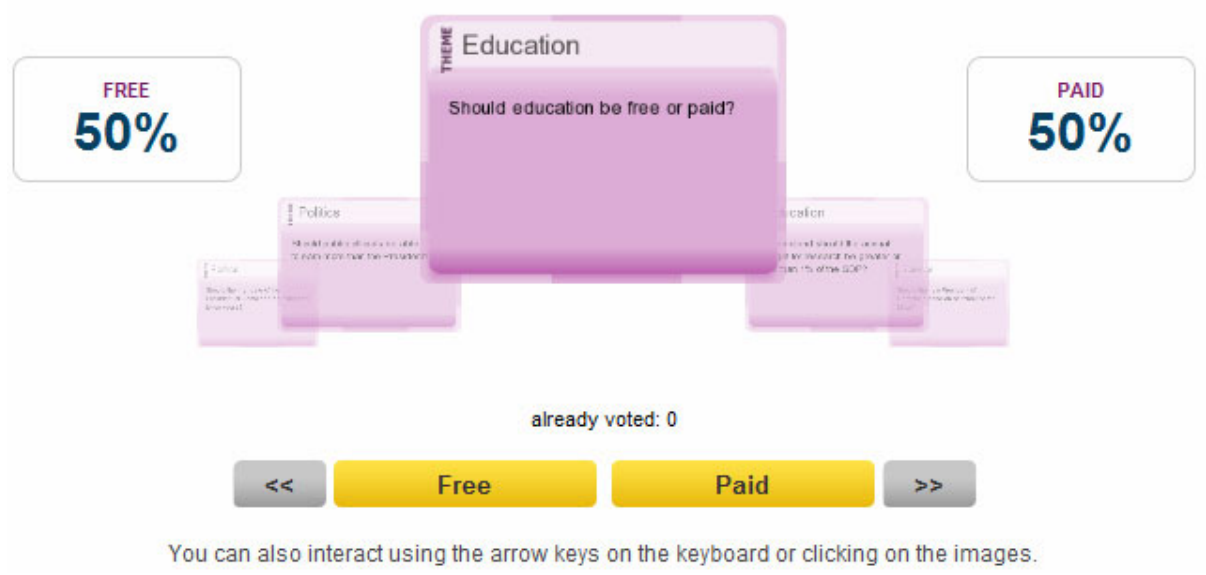

Fig. 2. Interface used for citizen surveys

In the setting where users are required to register to vote, each user can only vote once, but it is always possible change the vote. The main purpose of this section is to know the community opinion in relation to key topics.

\subsection{The Candidates`Electoral Program}

The fourth main area of the proposed platform offers the citizen the means to compare side-by-side the different candidates` electoral programs, and the candidate with an additional channel to broadcast the political messages to the electorate.

If iLeger is configured to be used directly by the candidates, in this section they can introduce via the Web interface the individual proposals in a given topic, e.g. Economy or Health Care. These proposals are then voted and commented by the citizens directly on the platform. Predicting cases of low-ranked proposals, the platform was designed such that the candidates can easily re-write or even remove unpopular entries. This area has the potential to create an interactive process for drafting a candidate's program taking into account direct feedback from the community. Moreover, the candidates can publish their comments to the other candidate's proposals, possibly for identifying their disadvantages and weaknesses, enriching therefore the debate around the electoral programs. With this debate and discussion around the political issues at hand the citizen can have a better idea of the 
position taken by each candidate, not only based on their own program but also by the contributions in the debate around the political position taken.

If iLeger is configured without the direct interaction of candidates, this section can be used to publish the editorial version of the electoral programs for the various candidatures structured by topics, and to enable citizens to issue their opinion by voting and by commenting on the various measures proposed by the candidatures. Naturally, with this configuration the debates between candidatures and between candidatures and citizens are lost.

In addition, as a direct consequence of the compilation of all electoral programs from the candidates, they can easily be held accountable after the elections since a clear list of proposals will be available for monitoring. This also opens the way to future use of the content in this section after the electoral period.

\subsection{Live Debates}

In this area of iLeger, users can read archived discussions, gather information about scheduled debates, and if there is a live debate ongoing, they can access and participate in this debate. To enable the live debates this section incorporates the component CoveritLive (http://www.coveritlive.com/).

Throughout an election period several live debates with the candidatures may be conducted. As mentioned previously, the discussions are moderated and will not require that citizens $\log$ in to submit questions and comments.

One of the innovative features of the iLeger consists of the use of the section dedicated to questions and suggestions for the creation of TOPs that can then be used during the live debates. With the purpose of creating these TOPs, the citizens are initially invited to submit questions and suggestions and vote on their relevance for a predetermined period of time. Then, along with the statistical information collected in the section dedicated to citizen surveys, the TOP questions and suggestions may be used to feed the live debates.

Therefore, even if iLeger is configured without direct interaction by the candidatures in the other sections, it can still be used in such a way that the candidatures can be invited to participate sporadically in live discussions with citizens. This feature can be useful for situations in which the candidatures do not want to commit themselves for longer periods of time due, for example, to scarcity of resources. In this scenario, iLeger assumes then the role of technological support for an editorial user.

\section{Case Study: The 2011 Portuguese Presidential Elections}

iLeger was used in partnership with SAPO, the largest Portuguese Web portal, during the Portuguese Presidential Elections held in January of 2011. It covered the last two weeks before the elections. All the six candidatures were invited to join and participate in iLeger and all of them accepted.

The version of iLeger used in these elections was configured so that users had to be registered in order to submit contents and vote, all written content submitted by 
citizens were subject to moderation and representatives of the different candidatures only participated in live debates.

For user registration, the single sign on (SSO) mechanism from our partner was used. This way, users already registered on SAPO could log in to iLeger without the need for new registration. We recorded 947 distinct users who have logged in, that is, who were enabled to submit written content and vote. Statistics extracted from Google Analytics showed that during the two weeks 23,512 unique users visited iLeger (total of 62,306 page views). The logged in to (unique) visitors ratio amounts therefore to $4 \%$.

During the first week, citizens were asked to create a Top 10 questions to be posed to the representative of each candidature in live debates held in the second week, the last before the election. To this end, users submitted questions and voted for their relevance. At this stage, were accepted by the moderator 187 of the 253 questions posed by users, indicating a rejection rate of $26 \%$. In addition, there were submitted 48 comments around some of the questions, indicating a low level of debate. These results are in line with others found in the literature [18].

In the second week six live debates were conducted, one with each candidature. Each debate lasted an hour and a half. It is important to note that from the 23,512 unique visitors over the two weeks, 9862 entered iLeger for the first time during these debates, demonstrating the interest of citizens to participate in live events of short duration.

During the live debates, the Top questions generated during the first week were asked to the representatives of the candidatures, with additional questions submitted by citizens during the live debates. Over the six debates there were 972 entries submitted in the form of questions or comments. However, by restrictions of time and moderation, only 93 of these entries were addressed by candidatures.

Over the two weeks the registered users submitted a total of 201 suggestions, of which 20 were rejected by the moderator. In this section of iLeger, the users were encouraged to say what they would do if they were President of Portugal. Overall there were 886 votes on questions, 1292 votes on suggestions and 6265 votes on the 12 questions of the survey.

It is also interesting to note that from the 947 users who have logged in, only 251 have submitted written content (253 questions, 201 suggestions and 48 comments), showing a percentage of $26.5 \%$. Moreover, considering the 23,512 unique users who visited $i$ Leger, then only $1 \%$ has submitted written content.

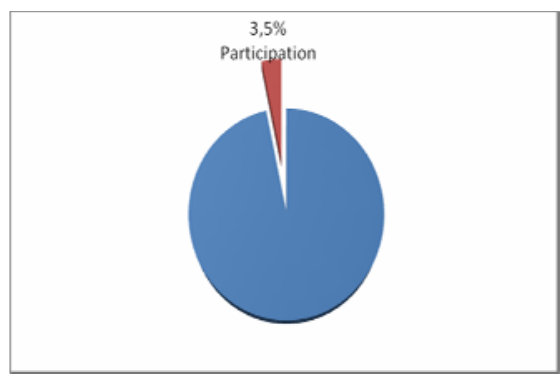

Fig. 3. Percentage of participation within unique visitors 
If, besides the introduction of written content, we account for the action of voting on the questions, suggestions and inquiries, we will then obtain 845 different users that actively interacted with iLeger. Following these measurements, as shown in Figure 3, we accounted for $3.5 \%$ of unique visitors actively participating in iLeger.

From Figure 4 it can be verified that $89 \%$ of the users that logged in, participated in iLeger.

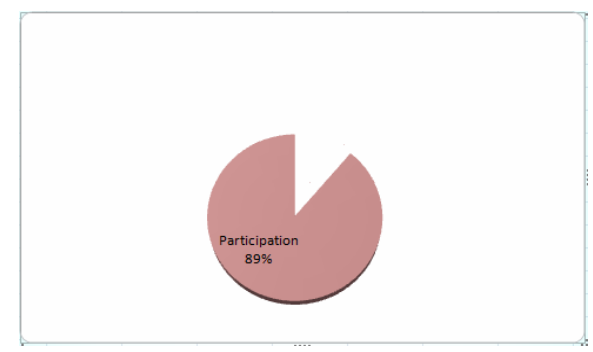

Fig. 4. Percentage of participation within logged in users

In these elections iLeger was primarily used to identify key issues and suggestions from voters, as well as their views on the key issues of the election. Through live debates, it was also possible to obtain a better insight of the viewpoint of representatives of the candidatures regarding the 10 questions most voted by the citizens in the previous week as well as other live questions. By comparing the viewpoints, the citizens will have additional information about the candidates' position on the important issues, helping them decide for their best candidate. However, due to the short time iLeger was online and, consequently, due to the limited amount of data gathered, it is still not possible to respond accurately to all research questions left open at the introduction of this paper.

\section{Future Work}

In a project as ambitious as this, there are certainly many improvements possible and many directions for future development and further research. It should be emphasized that this Web application is based on an incremental development process starting from a limited set of features, allowing for an early release date and for feedback already in the first stages of the project. In addition, from the user's perspective it is also advantageous since simpler and fewer functionalities are easier to learn. New functionalities can therefore be acquired, in an incremental way, as they are made available. The users themselves can provide their ideas and suggestions for new functionalities which will be taken into account when deciding the development path for the application.

Considering the continuous development of this electoral application, after more detailed consultation of the stakeholders (the citizens and the candidates), the main needed functionalities are workflow and staff management to the candidates, and management of favorite contributions to the citizens. It is also our intention to assess the quality of the platform. 
In addition, as a direct consequence of the application development and usage, the following open questions were identified for future research and development: What should be the role and scope of user moderation? How to implement self or mutual moderation mechanisms? How important is the role of an information curator in this context? How to improve usability and optimize information architecture? How to prevent duplicated entries? How to manage a possibly large amount of questions, proposals and historical data? ILeger takes several approaches to deal with this scalability problem. In order to keep the number of comments, questions and ideas manageable, the users are encouraged to vote on the existing questions or ideas instead of submitting repeated or re-phrased ones. Additionally, the content is categorized by topics providing navigation structure and organization to the data submitted.

Moreover, it is a subject for further investigation the perceived idea that citizens prefer to participate in events of short duration instead of ongoing a lasting discussion.

One important direction of future work concerns the technological support for live debates which, due to the general interface used, can be better tailored to this particular context. Another identified area for future work concerns the incorporation of comments in the section dedicated to citizen surveys to foster debate about the issues under discussion.

\section{Conclusion}

All candidates are well aware of the current momentum in Internet-based social networks and dedicate more and more financial and human resources for transmitting their messages across the electorate through Web channels such as Twitter and Facebook and their own websites.

From the experience gather during the 2011 Portuguese presidential election we can conclude that publicity is a key factor in this kind of initiatives. Every time our partner placed iLeger on their headlines the number of accesses increased enormously.

After observing how users interacted with iLeger, the statistics derived there from, and the overall reactions both from the citizens and the candidates contacted, we believe Internet-based tools to support eDemocracy such as this one will become mainstream. These digital tools would provide a major contribution to reverse the current disengagement from political and electoral debate, as well as provide a valuable means to bring together both citizens and politicians through open and direct dialogue - a dialogue with a digital memory open to future consultations. Moreover, we believe that continuous dialogue may also improve citizen trust and accountability of politicians, although more research is needed to confirm this assumption.

We are deeply convinced that iLeger can be an effective tool for elections 2.0, and we hope that it provide a new way of citizen-candidate interaction who can also inspire other initiatives for finding innovative solutions in eDemocracy. 


\section{References}

1. Creighton, J.L.: The Public Participation Handbook: Making Better Decisions Through Citizen Involvement. Jossey-Bass, San Francisco (2005)

2. Sanford, C., Rose, J.: Characterizing eParticipation. International Journal of Information Management 27, 406-421 (2007)

3. Putnam, R.D.: Bowling Alone: The Collapse and Revival of American Community. Simon \& Schuster, New York (2000)

4. Coleman, S., Blumer, J.: The Internet and Democratic Citizenship - Theory, Practice and Policy, pp. 166-197. Cambridge University Press, Cambridge (2009)

5. Chadwick, A., Howard, P.: New Directions in Internet Politics Research. In: Chadwick, A., Howard, P. (eds.) The Routledge Handbook of Internet Politics, pp. 1-9. Routledge Taylor \& Francis Group (2010)

6. Macintosh, A., Coleman, S., Schneeberger, A.: eParticipation: The Research Gaps. In: Macintosh, A., Tambouris, E. (eds.) ePart 2009. LNCS, vol. 5694, pp. 1-11. Springer, Heidelberg (2009)

7. ParticipateDB, http://www.participatedb.com/projects (accessed March 13, 2011)

8. Panopoulou, E., Tambouris, E., Tarabanis, K.: eParticipation Initiatives in Europe: Learning from Practitioners. In: Tambouris, E., Macintosh, A., Glassey, O. (eds.) ePart 2010. LNCS, vol. 6229, pp. 54-65. Springer, Heidelberg (2010)

9. Seelye, K.: Asked Millions Reply. The Caucus - The Politics and Government Blog of the Times. New York Times,

http: / / www . thecaucus.blogs.nytimes.com/2008/10/06/

asked-millions-reply/ (accessed May 7, 2010)

10. Smith, A.: The Internet's Role in Campaign 2008. Pew Research Center (2009)

11. SmartVote, http: / / www. smartvote.ch/ (accessed March 15, 2011)

12. CNN ElectionCenter2008,

http: / / edition.cnn. com/ELECTION/2008/issues/(accessed May 6, 2010)

13. Abgeordneten, http: //www. abgeordnetenwatch. de/ (accessed May 20, 2010)

14. Taylor-Smith, E., Lindner, R.: Using Social Networking Tools to Promote eParticipation Initiatives. In: Prosser, A., Parycek, P. (eds.) Proceedings of EDEM 2009 - Conference on Electronic Democracy, Vienna, pp. 115-121 (2009)

15. Wright, S.: Government-run Online Discussion Fora: Moderation, Censorship and the Shadow of Control. British Journal of Politics and International Relations 8(4), 550-568 (2006)

16. Coleman, S., Gøtze, J.: Bowling Together - Online Public Engagement in Policy Deliberation. Information Polity 7(4), 247-252 (2002)

17. Tomkova, J.: E-consultations: New tools for civic engagement or facades for political correctness? European Journal of ePractice 7, 45-54 (2009)

18. Aichholzer, G., Westholm, H.: Evaluating eParticipation Projects: Practical Examples and Outline of an Evaluation Framework. European Journal of ePractice 7, 27-44 (2009) 